\title{
Fuzzy Identity-Based Threshold Key-Insulated Encryption with Ciphertext Policy
}

\author{
Jianhong Chen ${ }^{1, a}$ \\ ${ }^{1}$ Faculty of Computer and Software Engineering, Huaiyin Institute of Technology, Huaian 223003, \\ China
}

a1395996433@qq.com

\begin{abstract}
Keywords: Threshold key-insulated; fuzzy identity based; encryption; ciphertext policy.
Abstract. To solve the signing key exposure problem in fuzzy identity-based encryption systems with ciphertext policy, we propose a fuzzy identity-based threshold key-insulated encryption scheme with ciphertext policy (FIBTKIE-CP) which is provably secure. Our scheme is key-insulated and strongly key-insulated. Even if temporary private keys for up to $N-1$ time periods are compromised, an adversary is still unable to obtain this user's temporary private key from the remaining time period. Even if up to $k-1$ helpers are exposed and all temporary private keys are compromised, the adversary still can not harm the security of the non-exposed periods..
\end{abstract}

\section{Introduction}

Security is harmed by inadvertent loss of private keys. In 2002, Dodis et al. [3] introduced a key insulation mechanism, which can protect secret keys in public key cryptosystems. Weng et al. [5] proposed the threshold key-insulation in which for at least $k$ out of $n$ helpers are used to refresh the user's temporary private keys. Ciphertext policy FIBE (FIBIE-CP) [2] is a variant of FIBE [4]. In a FIBIE-CP system, attributes are associated with user secret keys and access structures with ciphertexts. To deal with the key exposure problem in FIBE systems, Chen et al. gave a fuzzy identity-based parallel key-insulated encryption (FIBPKIE-CP) [1] scheme. But Chen et al. used two different helpers to refresh the private keys. There are some scenarios in which at least $k$ out of $n$ helpers are needed to update the user's temporary private keys. To strengthens the security and flexibility of Chen et al.'s scheme, we give a fuzzy identity-based threshold key-Insulated encryption scheme with ciphertext policy (FIBTKIE-CP) in which decryption is enabled if and only if the user's identity (attribute set) satisfies the access structure.

\section{Model of FIBTKIE-CP}

\section{Definition}

Throughout this paper, we use bilinear pairings, DBDH assumption and PRF[1]. We let $Z_{p}^{*}$ denote the set $\{0,1,2, \ldots, p-1\}$ and denote $Z_{p} / 0$. For a finite set $S, x \in{ }_{\mathrm{R}} S$ means choosing an element $x$ from $S$ with a uniform distribution. A FIBTKIE-CP scheme consists of six algorithms:(1)Setup( $\kappa)$ : Given a security parameter $\kappa$, the authority runs this algorithm to output a master secret key msk and a public key $p k ;(2) \operatorname{KeyGen}(\omega, m s k)$ : Given the user's identity $\omega$, as a set representing a user's attributes, and the master-key $m s k$, the authority runs this algorithm to output an initial private key $T K_{\omega, 0}$ and $n$ helper keys $\left\{H K_{\omega, i^{\prime}}\right\}_{1 \leq i^{\prime} \leq n}$ corresponding to $\omega$. Each helper key $H K_{\omega, i^{\prime}}$ is kept by the $i^{\prime}$-th helper and the user with identity $\omega$ keeps the initial private key. (3)HelperUpt $\left(t, \omega, H K_{\omega}, p k\right)$ : The helper key-update algorithm takes as input a period index $t$, an identity $\omega$ and his $i^{\prime}$-th $\left(1 \leq i^{\prime} \leq n\right)$ helper key $H K_{\omega, i^{\prime}}$. It outputs the $i^{\prime}$-th key-update information share $U I_{\omega, t, i^{\prime}}$ with respect to identity $i^{\prime}$ and period $t$. (4) UserUpt $\left(t, \omega, T K_{\omega, t}, U I_{\omega, t^{\prime}, t}, P K\right)$ : The user key-update algorithm takes as input an identity $\omega$, his temporary private key $T K_{\omega, t^{\prime}}$ for period $t^{\prime}$, and a set $\left\{U I_{\omega, t, i^{\prime}}\right\}_{i^{\prime} \in S^{\prime \prime}}$ of key-update information shares, where $S \subseteq\{1, \ldots, n\}$ and $\left|S^{\prime}\right| \geq k$. It returns this user's temporary private key $T K_{\omega, t}$ for period $t$, and deletes $T K_{\omega, t^{\prime}}$ and $\left\{U I_{\omega, t, i^{\prime}}\right\}_{i^{\prime} \in S^{\prime \prime}} ;(5)$ Encryption $(t, M, W, p k)$ : The Encryption algorithm takes as input the public key $p k$, the time period index $t$, a message $M$ and an access structure $W$. It returns a ciphertext 
$(t, E)$ such that a temporary private key generated from attribute set $\omega$ for period $t$ can be used decrypt $(t, E)$ if and only if $\omega \mid=W ;(6)$ Decryption $\left(t, E, \omega, T K_{\omega, t}, p k\right)$ : The Decryption algorithm takes as input a ciphertext $(t, E)$ and a temporary private key $T K_{\omega, t}$. It returns the message $M$ if $\omega$ satisfies $W$, where $S$ and $t$ are the identity (attribute set) and the time period index respectively used to generate $T K_{\omega, t}$.

\section{Security notions for FIBTKIE-CP}

A FIBTKIE-CP scheme is said to be secure against chosen plaintext attacks (CPA) in the sense of key-insulation if no probabilistic polynomial-time adversaries have non-negligible advantage in the following game. For convenience, we give the definition of a restricted identity as below: the attribute set of the restricted identity satisfies challenge access structure $W^{*}$.

Init. The adversary declares the access structure $W^{*}$ and the time period index $t^{*}$ that he wishes to be challenged upon.

Setup.The challenger runs the setup phase of the algorithm and tells the adversary the public parameters.

Phase 1. The adversary adaptively issues a set of queries as below:(1)Key Generation Query $\langle\gamma\rangle$ : The challenger first runs algorithm KeyGen to obtain the initial private key $T K_{\gamma, 0}$ and $n$ helper keys $\left\{H K_{\gamma, i^{\prime}}\right\}_{1 \leq i^{\prime} \leq n}$. It then sends these results to the adversary; (2) Helper Key Query $\left\langle\gamma, i^{\prime}\right\rangle$ : The challenger responds by running algorithm KeyGen to generate $H K \gamma, i$ and sends it to the adversary; (3)Temporary Private Key Query $\langle\gamma, t\rangle$ : The challenger responds by running algorithms HelperUpt and UserUpt to generate $T K_{\gamma, t}$. It then returns it to the adversary.

Challenge. The adversary submits two equal length messages $M_{0}, M_{1}$. The challenger flips a random coin, $b$, and encrypts $M_{b}$ with $W^{*}$ and $t^{*}$. The ciphertext is passed to the adversary.

Phase 2. Phase 1 is repeated.

Guess. The adversary outputs a guess $b^{\prime}$ of $b$.

The advantage of an adversary $A$ in this game is defined as $\operatorname{Pr}\left[b^{\prime}=b\right]-1 / 2$. We refer to the above game as an IND-FIBTKIE-CP-KI-CPA game. In the above game, it is mandated that the following conditions are simultaneously satisfied: (1) $A$ is disallowed to issue key generation queries for the restricted identities; (2) $A$ is disallowed to issue temporary private key queries for the restricted identities and the challenged time period $t^{*}$; (3) $A$ can only corrupt up to $k-1$ helper keys with respect to the restricted identities.

FIBTKIE-CP scheme is said to be secure against chosen plaintext attacks (CPA) in the sense of strong key-insulation if no probabilistic polynomial-time adversaries have non-negligible advantage in an IND-FIBTKIE-CP-SKI-CPA game. The IND-FIBTKIE-CP-SKI-CPA game is almost the same as the IND-FI\&KI-CPA game except Phase 1.

Phase 1. The adversary adaptively issues a set of queries as below:(1) Key Generation Query $\langle\gamma\rangle$ : the same as the IND-FIBTKIE-CP-KI-CPA game; (2) Helper Key Query $\left\langle\gamma, i^{\prime}\right\rangle: \mathrm{T}$ the same as the IND-FIBTKIE-CP-KI-CPA game.

The advantage of an adversary $A$ in this game is defined as $\operatorname{Pr}\left[b^{\prime}=b\right]-1 / 2$. In the above game, it is mandated that the following condition is satisfied: $A$ is disallowed to issue key generation queries for the restricted identities.

\section{Model of FIBTKIE-CP}

\section{Description of Our Scheme}

Our proposed FIBTKIE-CP scheme is based on Cheung-Newport's construction [2]. Let $G_{1}$ and $G_{2}$ be two groups with prime order $q$ of size $k, g$ be a random generator of $G_{1}$, and $e$ be a bilinear map such that $e: G_{1} \times G_{1} \rightarrow G_{2}$. Let $H$ be a collision-resistant hash function such that $H:\{0,1\}^{*} \rightarrow\{0,1\}^{n_{u}}$. We use a PRF family $F$ such that given a $\kappa$-bit seed (index) $s$ and a $\kappa$-bit argument (input) $x$, it outputs a $\kappa$-bit string $F_{s}(x)$. An access structure on attributes is a rule $W$ that returns either 0 or 1 given an identity $S$ (a set of attributes). We say that $S$ satisfies $W$ (written $S \mid=W$ ) if and only if $W$ answers 1 on $S$. Let the set of attributes be $N=\{1, \ldots, n\}$ for some natural number $n$. We regard attributes $i$ and their 
negations $\neg i$ as literals. We consider access structures that consist of a single AND gate whose inputs are literals. Let $W=\wedge i \in \underline{i}$ where $I \subseteq N$ and every $\underline{i}$ is a literal (i.e., $i$ or $\neg i$ ).

-Setup: The authority picks $y, t_{1}, \ldots, t_{3 n} \in{ }_{\mathrm{R}} Z_{p}, g_{2}, h_{1} \in \in_{\mathrm{R}} G_{1}$, sets $Y=e(g, g)^{y}$ and $T_{k}=g^{t_{k}}$ for each $k \in$ $\{1, \ldots, 3 n\}$. We define $H_{w}: Z_{p} \rightarrow G_{1}$ to be the function $H_{w}(x)=g_{1}^{x} h_{1}$. The public key is $p k=\left(G_{1}\right.$, $\left.G_{2}, e, g, g_{1}, Y, h_{1}, T_{1}, \ldots, T_{3 n}, H_{w}\right)$. The master secret key is $m s k=\left(y, t_{1}, \ldots, t_{3 n}\right)$. As illustrated in Table 1 , the public key elements $T_{i}, T_{n+i}$ and $T_{2 n+i}$ correspond to the three types of occurrences of $i$ : positive, negative and don't care.

Table 1.Common Parameters

\begin{tabular}{|c|c|c|c|c|c|}
\hline & 1 & 2 & 3 & $\ldots$ & $n$ \\
\hline positive & $T_{1}$ & $T_{2}$ & $T_{3}$ & $\ldots$ & $T_{n}$ \\
\hline negative & $T_{n+1}$ & $T_{n+1}$ & $T_{n+3}$ & $\ldots$ & $T_{2 n}$ \\
\hline Don't Care & $T_{2 n+1}$ & $T_{2 n+1}$ & $T_{2 n+3}$ & $\ldots$ & $T_{3 \mathrm{n}}$ \\
\hline
\end{tabular}

-KeyGen: To generate the helper key and the initial private key for identity $S$, the authority does as follows. Let $S$ denote the input identity (attribute set). Every $i \in S$ is implictly considered a negative attribute. Pick $r_{i} \in{ }_{\mathrm{R}} Z_{p}$ for every $i \in N$ and set $r=\sum_{i=1}^{n} r_{i}$. Randomly choose a helper key $H K_{S} \in \mathrm{R}\{0,1\}^{\mathrm{\kappa}}$, compute $k_{S, 0}=F_{H K_{S}}(0)$. Note that if the length of the input for $F$ is less than $\kappa$, we can add some "0"s as the prefix to meet the length requirement. Let $\hat{D}_{S, 0}^{\prime}=g^{y-r} H_{w}(0)^{k_{S, 0}}, \hat{D}_{S, 0}^{\prime \prime}=g^{k_{S, 0}}$. For each $i \in N$, let $D_{i}=$ if $i \in S$; otherwise, let $D_{i}=g^{\frac{r_{i}}{t_{n+i}}}$. Let $F_{i}=g^{\frac{r_{i}}{t_{2 n+i}}}$ for every $i \in N$. Pick $\beta \in \mathrm{R} Z_{p}^{*}$ and set $R=g^{\beta}$, compute the initial private key $T K_{S, 0}=\left(R,-,-,\left\{D_{i}\right\}_{i \in N},\left\{F_{i}\right\}_{i \in N}\right)$.

For each $i \in \omega$ and each index $i^{\prime} \in\{1, \ldots, k-1\}$, pick $l_{i, i^{\prime}} \in \in_{\mathrm{R}} Z_{p}^{*}$ and set the $i^{\prime}$-th helper key to be

$H K \omega, i^{\prime}=\left(\left\{H K_{i, i^{\prime}}\right\}_{i \in \omega)}=\left(\left\{g_{2}^{l_{i, j}}\right\}_{i \in \omega}\right)\right.$

Let $S^{\prime}=\{0,1, \ldots, k-1\}$. For each $i \in \omega$ pick $s_{i} \in{ }_{\mathrm{R}} Z_{p}^{*}$. For each remaining index $i^{\prime} \in\{k, \ldots, n\}$, set the $i^{\prime}$-th helper key to be

$\left(\left\{\left(g_{2}^{y-r-\beta}\right)^{\Lambda_{i, S^{\prime}}(0)}\left(\prod_{j=1}^{k-1} H K_{i, i^{\prime}}\right)^{\Lambda_{, S^{\prime}(j)}}\right\}_{i \in \omega}\right)$

-HelperUpt: Given a period index $t$, an identity $\omega$ and his $i^{\prime}$-th $\left(1 \leq i^{\prime} \leq n\right)$ helper key $H K_{\omega, i^{\prime}}$, this

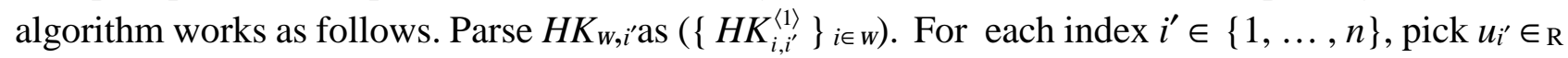
$Z_{p}^{*}$ and output user $\omega^{\prime}$ 's $i^{\prime}$-th key-update information share $U I_{\omega, t, i^{\prime}}$ for period $t$ as

$$
\begin{aligned}
U I_{\omega, t, i^{\prime}} & =\left(\left\{H K_{i, i^{\prime}} H_{w}(t)^{u_{i}}\right\}_{i \in \omega}, g^{u_{i^{\prime}}}\right) \\
& =\left(\left\{g_{2}^{l_{i, j}} V(i)^{r_{i, r^{\prime}}} H_{w}(t)^{u_{i}}\right\}_{i \in \omega, g^{u_{i}}}\right)
\end{aligned}
$$

-UserUpt: Given an identity $\omega$, a temporary private key $T K_{\omega, t^{\prime}}$ for period $t^{\prime}$, and a set $\left\{U I_{\omega, t, i^{\prime}}\right\}_{i^{\prime} \in S}$ of key-update information shares for period $t$, where $S^{\prime \prime} \subseteq\{1, \ldots, n\}$ and $\left|S^{\prime \prime}\right| \geq k$ (for convenience, we assume $\left.\left|S^{\prime \prime}\right|=k\right)$, this algorithm works as follows. Parse $T K_{\omega, t^{\prime}}$ as $(R$, $\left.\hat{D}_{S, t^{\prime}}^{\prime}, \hat{D}_{S, t^{\prime}}^{\prime \prime},\left\{D_{i}\right\}_{i \in N},\left\{F_{i}\right\}_{i \in N}\right)$; Parse $U I_{\omega, t, i^{\prime}}$ as $\left(U I_{i, t, i^{\prime}}^{\langle 1\rangle}, U I_{i, t, i^{\prime}}^{\langle 2\rangle}\right)$; Set user $\omega^{\prime}$ 's temporary private key $T K_{\omega, t}$ for period $t$ to be $\left(R,\left(\prod_{i^{\prime}} U I_{i, t, i^{\prime}}^{\langle 1\rangle}\right)^{\Delta_{i, S^{\prime}}(0)},\left(\prod_{i^{\prime}} U I_{\omega, t, i^{\prime}}^{\langle 2\rangle}\right)^{\Delta_{i, S^{\prime}}(0)},\left\{D_{i}\right\}_{i \in N,},\left\{F_{i}\right\}_{i \in N}\right)$. Note that in time period $t$, if let $u=\sum_{i^{\prime} \in S^{\prime}} \Delta_{0, s^{\prime}}\left(i^{\prime}\right) \cdot u_{i^{\prime}}$, then $T K_{\omega, t}$ is always set to be

$$
\left(R, \hat{D}_{S, t}^{\prime}, \hat{D}_{S, t^{\prime}}^{\prime \prime},\left\{D_{i}\right\}_{i \in N},\left\{F_{i}\right\}_{i \in N}\right)=\left(g^{\beta}, \quad\left\{g^{y-r-\beta} H_{w}(t)^{u}\right\}_{i \in \omega}, g^{u},\left\{D_{i}\right\}_{i \in N},\left\{F_{i}\right\}_{i \in N}\right) \text {. }
$$


-Encryption: Given time period index $t$, a message $M \in G_{1}$ and an AND gate $W=\wedge_{i \in I} \underline{i}$, this algorithm does as follows. Pick $s \in{ }_{\mathrm{R}} Z_{p}$; For each $i \in I$, let $E_{i}=\mathrm{T}_{\mathrm{i}}^{\mathrm{s}} \quad$ if $\underline{i}=i$ and $\mathrm{T}_{\mathrm{n}+\mathrm{i}}^{\mathrm{s}}$ if $\underline{i}=\neg i$; for each $i \in M I$, let $E_{i}=$ $\mathrm{T}_{2 \mathrm{n}+\mathrm{i}}^{\mathrm{s}}$. The ciphertext is $\quad(t, E)=\left(t,\left(W, E^{\prime}=M \cdot Y^{s}, E^{\prime \prime}=g^{u}, E^{\prime \prime \prime}=H_{w}(t)^{u},\left\{E_{i}\right\}_{i \in N}\right)\right)$

-Decryption: Suppose the input ciphertext is of the form $(t, E)=\left(t,\left(W, E^{\prime}, E^{\prime \prime}, E^{\prime \prime \prime},\left\{E_{i}\right\}_{i \in N}\right)\right)$, where $W$ $=\wedge_{i \in I} \underline{i}$. Also, let $\omega$ denote the identity used to generate the input secret key $T K_{\omega, t}=\left(g^{\beta}\right.$, $\left.\left\{g^{y-\bar{r}-\beta} H_{w}(t)^{u}\right\}_{i \in \omega}, g^{u},\left\{D_{i}\right\}_{i \in N},\left\{F_{i}\right\}_{i \in N}\right)$. For each $i \in I$, this algorithm computes the pairing $e\left(C_{i}, D_{i}\right)$. If $\underline{i}=i$ and $i \in \omega$, then $e\left(E_{i}, D_{i}\right)=e\left(g^{t_{i} \cdot s}, g^{\frac{r_{i}}{t_{i}}}\right)=e(g, g)^{r_{i} \cdot s}$; If $\underline{i}=\neg i$ and $i \in \omega$, then $e\left(E_{i}, D_{i}\right)=e\left(g^{t_{n+i} \cdot s}, g^{\frac{r_{i}}{t_{n+i}}}\right)$ $=e(g, g)^{r_{i} \cdot s}$; for each $i \in I$, this algorithm computes the pairing $e\left(E_{i}, F_{i}\right)=e\left(g^{t_{2 n+i} \cdot s}, g^{\frac{r_{i}}{t_{2 n+i}}}\right)=e(g, g)^{r_{i} \cdot s}$. Then, the ciphertext can be decrypted as

$$
\begin{aligned}
& M=\frac{E^{\prime} e\left(E^{\prime \prime \prime}, \hat{D}_{S, t}^{\prime \prime}\right)}{e\left(E^{\prime \prime}, R \cdot \hat{D}_{S, t}^{\prime}\right) \prod_{i=1}^{n} e(g, g)^{r_{i} \cdot s}}=\frac{M \cdot Y^{s} e\left(H_{w}(t)^{s}, g^{u}\right)}{e\left(g^{s}, g^{\beta} g^{y-r-\beta} H_{w}(t)^{u}\right) e(g, g)^{r \cdot s}} \\
& =\frac{M \cdot Y^{s} e\left(H_{w}(t)^{s}, g^{u}\right)}{e\left(g^{s}, g^{y-r}\right) e\left(g^{s}, H_{w}(t)^{u}\right) e(g, g)^{r \cdot s}}=\frac{M \cdot Y^{s}}{e(g, g)^{y \cdot s}}=\frac{M \cdot Y^{s}}{Y^{s}}
\end{aligned}
$$

\section{Security}

The proof of our proposed FIBTKIE-CP scheme is similar with that of Chen et al.'s FIBPKIE-CP[1].

\section{Conclusions}

We introduce the notion of fuzzy identity-based key-insulated encryption with ciphertext policy (FIBTKIE-CP) and describe a construction that is provably secure.

\section{References}

[1] J. Chen, K. Chen, Y. Long , Z. Wan , K. Yu, C. Sun and L. Chen: Ciphertext Policy Attribute-Based Parallel Key-Insulated Encryption. in: Journal of Software, Vol. 23, No. 10, (2012), p. 2795-2804

[2] L. Cheung and L. Newport: Provably Secure Ciphertext Policy ABE. in: Proceedings of ACM Computer and Communications Security (CCS), (2007), p. 456-465

[3] Y.Dodis, J. Katz, S. Xu and M. Yun: Key-Insulated Public-Key Cryptosystem. in: Proceedings of International Conference on Theory and Applications of Cryptographic Techniques (Eurocrypt), (2002), p. 65-82

[4] A. Sahai and B. Waters: Fuzzy Identity-Based Encryption. in: Proceedings of International Conference on Theory and Applications of Cryptographic Techniques (Eurocrypt), (2005), p. 457-473

[5] J. Weng, X. Li, K. Chen and S. Liu: Identity-Based Threshold Key-Insulated Encryption without Random Oracles. in: Proceedings of International Conference on the Cryptographers' Track at the RSA (CT-RSA), (2008), p. 203-220 